\title{
Revisión de las técnicas de carga cognitiva inducida en el ámbito de la detección de la mentira
}

Review of induced cognitive loading techniques in the field of lie detection.

\section{Silvestre Cabezas García ${ }^{1}$}

(1) Fundacion Universitaria Behavior \& Law, Madrid, España.

Email correspondencia: cabezas@behaviorandlaw.com

\section{Resumen}

El presente artículo trata de profundizar en el campo de la detección de mentiras haciendo un repaso de los enfoques tradicionales que se han llevado a cabo en este campo para pasar después a una perspectiva más moderna basada en la carga cognitiva inducida. Para ello, se hace una revisión de varios estudios publicados en los últimos años sobre inducción de carga cognitiva y detección de la mentira, analizando las técnicas empleadas y los resultados de cada una. Finalmente se discute la efectividad de estas, llegando a la conclusión de que no suponen una mejora sustancial a las técnicas tradicionales, si bien es cierto que al ser más recientes requieren más estudios y tienen mayor potencial.

Palabras Clave: Carga cognitiva; detección de mentiras; técnicas de detección; psicología cognitiva.

\begin{abstract}
The present paper tries to deepen the field of lie detection by reviewing the traditional approaches that have been carried out in this field and then move to a more modern perspective based on induced cognitive load. To this end, a review is made of several studies published in recent years on induction of cognitive load and detection of lies, analyzing the techniques used and the results of each one. Finally, the effectiveness of these techniques is discussed, reaching the conclusion that they do not represent a substantial improvement to traditional techniques, although it is true that as they are more recent, they require more studies and have greater potential.
\end{abstract}

Keywords: Cognitive load; lie detection; detection techniques; cognitive psychology.

Fecha de envío: 14/10/2019

Fecha de aceptación: 30/11/2019 


\section{INTRODUCCIÓN}

Los seres humanos tenemos una capacidad innata para mentir que es parte de la naturaleza humana (Valverde, Ruiz y Llor, 2013). Tradicionalmente se enseña que mentir es algo malo e indeseable que debe ser evitado (Bond y DePaulo, 2006) sin embargo, esto no parece ser un gran problema y las personas no muestran unas grandes dificultades éticas para mentir cuando se les pide (Masip y Garrido, 2010). Lo que sí que es obvio es que la mentira y el engaño es algo con lo que convivimos día a día. Si esta se da en determinado ámbito puede no tener apenas consecuencias, como un niño cuando le dice a su madre que no ha sido él quien ha roto el jarrón. Sin embargo, existen otros ámbitos como el médico-pericial o el empresarial donde engañar puede suponer la pérdida de miles de euros.

Esto no tendría mayor trascendencia si los humanos fuésemos buenos detectores de engaño, pero lo cierto es que no es así. No sólo no es que no seamos buenos en la detección, sino que además los criterios que creemos fiables no lo son (Masip, 2005; Vrij. 2008). Los metaanálisis en este ámbito indican que no somos muy buenos discriminando entre verdades y mentiras (Bond y DePaulo, 2006; Hauch, Blandón-Gitlin, Masip y Sporer, 2015; Vrij, 2008). A pesar de los esfuerzos realizados, es difícil encontrar una fórmula mágica que discrime al 100\% las verdades y las mentiras y sea capaz de determinar con exactitud la veracidad de un testimonio sin ninguna duda. De hecho, diversas revisiones parecen apuntar a que son limitados los indicadores verbales, no verbales o fisiológicos relacionados de modo indudable con el engaño (Ben-Shakhar y Elaad, 2003; DePaulo et al., 2003; Kleiner, 2002; MacLaren, 2001; Masip y Alonso, 2006; Masip, Sporer, Garrido y Herrero, 2005; Vrij, 2000).

Debido a ello se han desarrollado múltiples métodos de detección y evaluación del engaño, principalmente centrado en tres vertientes: el de las medidas psicofisiológicas, el del contenido verbal y el de la conducta no verbal (Ovejero, 2009; Valverde et al., 2013), siendo el análisis de contenido verbal el que mejores resultados ha tenido en los últimos años. Por ello, se van a ver algunas de estas técnicas utilizadas en esta línea de investigación para luego centrarnos en el enfoque cognitivo de la detección de mentiras.

\section{A. Técnicas tradicionales de análisis de contenido verbal}

1) Evaluación de la Validez en las Declaraciones (Statement Validity Assessment) y Análisis de Contenido Basado en Criterios (Criteria Based Content Analysis)

El CBCA es el elemento central de un procedimiento más amplio denominado Evaluación de la Validez en las Declaraciones (Statement Validity Assessment, SVA), una de las técnicas más empleadas para la evaluación de la veracidad de las declaraciones verbales (Bensi, et al., 2009; Godoy-Cervera e Higueras, 2005; Vilariño, Formosinho y Cardoso, 2012; Vrij, 2000). El SVA es un procedimiento de generación y falsación de hipótesis sobre el origen de una declaración (Köhnkem. Manzanero y Scott, 2015), donde lo importante es analizar la credibilidad del contenido de la declaración y no realizar una valoración sobre la credibilidad o no de la persona (Steller y Köhnken (1989) y debe hacerse una vez se tenga el análisis conjunto (Ovejero, 2009).

El SVA se compone de tres fases:

1) Entrevista semiestructurada: se busca obtener la mayor cantidad de información de la manera menos sesgada posible y sin condicionar las respuestas del entrevistado (Garrido y Sobral, 2008; Jiménez, 2015). Su objetivo es obtener material sobre el cual aplicar el CBCA (Godoy-Cervera e Higueras, 2005).

2) Análisis de contenido usando el CBCA, del que se habla más adelante.

3) Listado de criterios de validez: tiene en cuenta distintos criterios que pueden influir en los resultados del CBCA (Godoy-Cervera e Higueras, 2005):

a) Características psicológicas. En esta categoría es importante evaluar la adecuación del lenguaje y el afecto y la susceptibilidad a la sugestión.

b) Características de la entrevista. El evaluador deberá realizar un análisis sobre la calidad de la entrevista valorando el tipo de preguntas formuladas (preguntas sugerentes, directivas o coactivas) y la adecuación global de la misma.

c) Motivación para realizar acusaciones falsas. Esta categoría pretende descartar aquellos aspectos de índole motivacional que pudieran estar influyendo para que la persona proporcione una declaración falsa. Un aspecto importante de esta categoría es hacer una valoración del contexto en el que se 
genera el informe.

d) Aspectos relacionados con la investigación. Este apartado está diseñado con el fin de valorar la consistencia entre las declaraciones e investigaciones previas y partes médicos o de otros tipos.

El CBCA se fundamenta en lo que se ha venido a denominar la hipótesis de Undeutsch (Steller, 1989) que postula que existe diferencia en cuanto a calidad y contenido entre declaraciones verdaderas e inventadas (Jiménez, 2015; Godoy-Cervera e Higueras, 2005) Consiste en 19 criterios con los que se analiza la entrevista una vez ha sido transcrita para ver si dichos elementos están presentes. Son criterios que tratan de identificar determinados contenidos que debería mostrar un relato que ha sido vivido realmente y que difícilmente aparecen en un relato inventado (Jiménez, 2015). Se analizan los criterios, si están presentes o no en la declaración y el grado de credibilidad dependerá del número y calidad de los criterios presentes (Sáiz, Baqués y Sáiz, 2006).

Tabla 1. Criterios de análisis del CBCA.

\begin{tabular}{|c|c|}
\hline $\begin{array}{l}\text { I. Características Generales } \\
\text { 1. Estructura lógica } \\
\text { 2. Elaboración no estrucutrada } \\
\text { 3. Cantidad de detalles }\end{array}$ & $\begin{array}{l}\text { II. Contenidos específicos } \\
\text { 4. Engranaje contextual } \\
\text { 5. Descripción de las interacciones } \\
\text { 6. Reproducción de la } \\
\text { conversación } \\
\text { 7. Complicaciones inesperadas } \\
\text { durante el accidente }\end{array}$ \\
\hline $\begin{array}{l}\text { III. Peculiaridades del } \\
\text { contenido } \\
\text { 8. Detalles inusuales } \\
\text { 9. Detalles superfluos } \\
\text { 10. Incomprensión de detalles } \\
\text { relatados con precisión } \\
\text { 11. Asociaciones externas } \\
\text { relacionadas } \\
\text { 12. Relatos del estado mental } \\
\text { subjetivo } \\
\text { 13. Atribución del estado mental } \\
\text { del agresor }\end{array}$ & $\begin{array}{l}\text { IV. Contenidos referentes a la } \\
\text { motivación } \\
\text { 14. Correcciones espontáneas } \\
\text { 15. Admitir falta de memoria } \\
\text { 16. Plantear dudas sobre el propio } \\
\text { testimonio } \\
\text { 17. Auto-desaprobación } \\
\text { 18. Perdón al autor de delito }\end{array}$ \\
\hline $\begin{array}{l}\text { V. Elementos específicos de la } \\
\text { ofensa } \\
\text { 19. Detalles característicos de la } \\
\text { ofensa }\end{array}$ & \\
\hline
\end{tabular}

Fuente: Jiménez (2015).

Vrij (2005) realizó un meta-análisis con 37 estudios sobre el CBCA y vio que los mejores criterios para discriminar eran cantidad de detalles, reproducción de conversaciones, engranaje contextual y elaboración inestructutrada; por el contrario, los peores fueron autodesaprobación y plantear dudas sobre el propio testimonio. La efectividad de identificación correcta de declaraciones verdaderas fue del 73\% (rango 53\%-89\%) y de declaraciones falsas del 72\% (rango 60\%-100\%). En estudios anteriores, Vrij et al. (2000; 2004a) encontraron que si utilizaban de forma conjunta indicadores no verbales e indicadores verbales del engaño (CBCA y RM) el porcentaje de clasificaciones correctas se incrementaba. Si bien es cierto que el CBCA tiene limitaciones (BlandónGitlin, Pezdek, Rogers y Brodie, 2005; Köhnken et al., 2015; Masip y Alonso, 2006; Vrij et al., 2005), la evidencia muestra que las declaraciones reales contienen más criterios de realidad que las declaraciones falsas (Gödert, Gamer, Rill y Vossel; 2005; Lee, Klaver y Hart, 2008; Vilariño, Novo y Seijo, 2011). Además, su uso combinado con otras técnicas mejora su precisión, que es justamente lo que hace el SAVE, unir la efectividad de distintas técnicas y protocolos. El propio Vrij menciona en CBCA como una herramienta valiosa para la investigación policial (Vrij et al., 2004b).

\section{2) Control de la Realidad (Reallity Monitoring)}

El Control de la Realidad parte de la idea de que las memorias de lo real y de lo imaginado varían en una serie de características (Ovejero, 2009; Vilariño et al., 2012. Por un lado, están los recuerdos externos que se generan a partir de la observación de un acontecimiento y por otro los recuerdos internos que son producto de nuestra imaginación (Sáiz et al., 2006; Valverde et al., 2013). Los recuerdos externos poseen mayor cantidad de información sensorial (detalles visuales, sonidos, olores y sensaciones físicas), más detalles contextuales (espacial y temporal) y menor referencias a procesos cognitivos que los externos (Alonso, 2009; Nahari, 2018; Ovejero, 2009; Sporer, 2004; Vrij, 2008).

Estos recuerdos pueden tener cuatro clases diferentes de información (Jiménez, 2015): a) aspectos sensoriales como olores, formas o sonidos; b) aspectos contextuales referidos al tiempo o momento y al espacio o lugar; c) aspectos semánticos; y d) operaciones cognitivas. A partir de ello se compone de ocho criterios para discriminar los relatos verdaderos de los falsos (Ovejero, 2009; Sáiz et al., 2006):

1. Claridad y viveza.

2. Detalles perceptuales.

3. Información espacial.

4. Información temporal.

5. Emociones y sentimientos.

6. Reconstrucción de la historia.

7. Realismo.

8. Operaciones Cognitivas.

Las evidencias muestran que cuando el relato es honesto se puntúa de manera positiva en 1, 2, 3, 4, 5, 6 y 7, 
y de manera negativa en el 8 (Valverde et al., 2013). Los criterios que más discriminan una declaración honesta son un mayor número de detalles perceptuales y de información espacial y temporal (Sáiz et al., 2006).

La eficacia de esta técnica es del 60-70\% aproximadamente (Vrij, 2008), siendo mucho mejor en la detección de sujetos honestos $(78,3 \%)$ que en la de mentirosos (35,3\%) (Lavoie, 2004; Sporer, 2004; Vrij, 2008; Vrij et al., 2004). Esta técnica tiene algunas ventajas respecto al $\mathrm{CBCA}$, como que requiere un entrenamiento más simple y tiene una base teórica más sólida (Masip et al., 2005; Ovejero, 2009).

\section{3) Uso estratégico de la evidencia (Strategic Use of Evidence)}

Esta técnica, tal como su nombre indica, se basa en el uso estratégico de la evidencia por parte del entrevistador. Evidentemente, para aplicar esta técnica es necesario tener primero algún tipo de evidencia que poder utilizar. Si se tiene, lo que ocurrirá, según Granhag, Strömwall y Hartwig (2007), es que los culpables tratarán de evitar mencionar información sobre esa evidencia, componiendo un relato en la declaración con incoherencias respecto a la evidencia del entrevistador, mientras que los inocentes serán más coherentes.

Las fases de la entrevista en base al SUE son (Granhag, Strömwall y Hartwig, 2007):

a) Planificación: el entrevistador busca información potencialmente incriminatoria que el sospechoso ignore que puede tener.

b) Recuerdo libre: se le pide que cuente qué hizo durante el tiempo que se cometió el delito.

c) Preguntas: se le formulan preguntas concretas, algunas referentes a esa información potencialmente incriminatoria.

d) Comprobación y compromiso: se repite y aclara la información, para comprobar que esté correcta y que se comprometa con ella.

e) Contraste entre la declaración y la evidencia incriminatoria: se le pide que explique las inconsistencias.

Harttwig, Granhag, Strömwall y Vrij (2005) mostraron que los culpables tendían a mencionar menos la información potencialmente incriminatoria en el recuerdo libre y más inconsistencias cuando la evidencia incriminatoria se mostraba al final. Estudios posteriores han mostrado una eficacia considerable en los aciertos a la hora de discriminar entre verdades y mentiras con un adecuado entrenamiento en esta técnica (Hartwig, Granhag, Strömwall y Kronkvist, 2006).

\section{B. Aproximación cognitiva a la detección de mentiras}

Un enfoque más reciente a los que se han descrito previamente es el cognitivo. Este enfoque que se enmarca proveniente de la Psicología Cognitiva se centra en una línea de investigación reciente que parte de la idea de que para mentir hace falta más esfuerzo mental que para decir la verdad (Walczyk, Mahoney, Doverspike y Griffith-Ross, 2009). De esta manera, adoptando el entrevistador un papel activo durante la entrevista, se puede aumentar el esfuerzo mental y las persona que mienten tendrán más dificultades para hacerlo; consecuentemente, las diferencias con los que dicen la verdad serán más notables. El núcleo de este enfoque es que los investigadores pueden magnificar las diferencias en las señales (no) verbales indicativas de la carga cognitiva mostrada por los que dicen la verdad de los mentirosos mediante intervenciones sobre principios cognitivos que hacen que la tarea de los mentirosos sea aún más exigente cognitivamente. Si tienen éxito, esas intervenciones deben dar como resultado que los mentirosos muestren más señales cognitivas de diagnóstico para el engaño y luego faciliten la detección de mentiras. El enfoque cognitivo de la detección de mentiras consiste en tres técnicas que pueden diferenciar a los que cuentan la verdad de los mentirosos: (1) hacer preguntas inesperadas, (2) alentar a los entrevistados a proporcionar más información e (3) imponer una carga cognitiva (Vrij et al., 2017).

\section{1) Preguntas inesperadas (Unanticipated Questions)}

Cuando dos o más sospechoso que son culpables se enfrenta a un interrogatorio en relación al hecho que han cometido, es muy probable que se esperen una serie de preguntas y se las preparen para dar las mismas respuestas. Sin embargo, si se les hacen preguntas inesperadas la coherencia entre las mismas desciende sustancialmente y permite discriminar entre culpables e inocentes. Vrij et al. (2009) encontraron diferencias significativas en preguntas espaciales, temporales y de dibujo en el plano; no encontraron diferencias en las respuestas a preguntas esperadas.

Algunos ejemplos de estas preguntas son (Vrij et al., 2009):

1. Dígame lo más detalladamente posible sobre el diseño del restaurante. 
Cabezas, S. (2019). Revisión de las técnicas de carga cognitiva inducida en el ámbito de la detección de la mentira. Behavior \& Law Journal, 5(1), 40-51.

2. ¿De qué temas hablaron durante el almuerzo? ¿En qué orden?

3. En relación con la puerta de entrada, ¿dónde se sentaron usted y su amigo?

4. En relación con la puerta de entrada y dónde se sentaron, ¿dónde estaban los comensales más cercanos?

5. En relación con la puerta de entrada, ¿en qué parte de la mesa se encontraba el camarero al servir su comida?

6. ¿Quién terminó primero de comer, usted o su amigo?

7. ¿Cuánto tiempo tomó entre que el camarero tomara su pedido y recibiera su comida?

2) Alentar a los entrevistados a dar más información (Encouraging interviewees to provide more information)

Aquellas personas que dicen la verdad suelen percibirse como más sinceras, dado que cuanta más información se de más probable es que se crean que el que habla dice la verdad (Johnson, 2006). Además, la información adicional que proporcionan aquellos que dicen la verdad podría dar pistas a los investigadores para verificar si es cierto o no lo que dice. A los mentirosos les puede resultar cognitivamente demasiado difícil agregar tantos detalles como hacen los verdaderos o, si los mentirosos agregan una cantidad suficiente de detalles, la información adicional puede ser de menor calidad o puede parecer menos plausible. También los mentirosos pueden ser reacios a agregar más información por miedo a que den pistas a los investigadores y, en consecuencia, no se crean sus mentiras. Esto significa que estas técnicas pueden conducir a que los verdaderos den más información y así se ha corroborado. La investigación experimental hasta el momento ha mostrado cuatro formas de facilitar que los que cuentan la verdad den más información: al utilizar un entrevistador que lo apoya, asintiendo con la cabeza y sonriendo durante una entrevista, dando un ejemplo de una respuesta modelo muy detallada (Bogaard, Meijer y Vrij, 2014), utilizando dibujos (Vrij, Mann, Leal y Fisher, 2012), y mediante el uso de la técnica de entrevista cognitiva (Ansarra et al., 2011; Colwell, Hiscock y Memon, 2002).

\section{3) Carga Cognitiva Inducida (Induced Cognitive Load)}

Partiendo de la misma premisa de que mentir es cognitivamente más complicado que decir la verdad se busca crear esa sobrecarga cognitiva con el fin de que así se evidencien señales observables (Masip y Herrero, 2015b). Imponer una carga cognitiva se refiere a las intervenciones de los investigadores destinadas a hacer que la entrevista sea más difícil mentalmente. Los mentirosos, que a menudo requieren más recursos cognitivos que quienes dicen la verdad en las entrevistas, tendrán menos recursos cognitivos. Si la demanda cognitiva aumenta aún más, lo que podría lograrse al hacer peticiones adicionales, los mentirosos pueden ser menos capaces que los que son sinceros para hacer frente a estas demandas adicionales (Vrij et al., 2017).

Una forma de causar esa sobrecarga cognitiva es mediante la técnica de orden inverso, donde se le pide a la persona que narre los hechos que ocurrieron, pero desde el final hacia el principio. Como puede apreciarse, de nuevo, el entrevistador adopta un papel activo. Lo que vieron los investigadores (Vrij et al., 2008; Vrij, Leal, Mann y Fisher, 2012) es que al realizar la narración en orden inverso modificaron su conducta otorgando mayores indicadores para discriminar entre verdades y mentiras.

También se ha conseguido hacer al instruir a los entrevistados a mantener contacto visual con el entrevistador (Vrij, Mann, Leal y Fisher, 2010) y pidiendo a los entrevistados que realicen dos tareas a la vez (VisuPetra, Varga, Miclea y Visu-Petra, 2013); o mediante turnos forzados que pueden emplearse cuando se entrevistan dos o más entrevistados al mismo tiempo (Vernham, Vrij, Mann, Leal y Hillman, 2014). Algunos meta-análisis posteriores han mostrado más eficacia del enfoque cognitivo de la evaluación de la credibilidad que del enfoque tradicional (Vrij, Fisher, Blank, Leal y Mann, 2016; Vrij. Fisher y Blank, 2017).

\section{Objetivos del trabajo}

El objetivo de esta revisión es conocer la situación actual en lo que respecta a la detección de la mentira en relación con las técnicas basadas en la teoría de la carga cognitiva, concretamente de la carga cognitiva inducida. Para ello se plantean dos objetivos:

- Determinar las técnicas usadas para aumentar la carga cognitiva.

- Analiza si estas técnicas basadas en el aumento de la carga cognitiva fueron eficaces para detectar la mentira.

\section{METODOLOGÍA}

\section{A. Materiales}

Se han seleccionado un total de 8 artículos científicos los cuales son estudios experimentales que se han centrado 
en el análisis de la detección de la mentira a partir del enfoque de la carga cognitiva usando una técnica para generar carga cognitiva inducida.

\section{B. Procedimiento}

Esta revisión se ha realizado a partir de la búsqueda de artículos científicos en las siguientes bases de datos: Web of Science, PsycInfo, Scopus, Medline y Psychology and Behavioral Sciences Collection.

Se realizó una búsqueda científica en estas bases de datos usando como palabras clave: “deception" y "cognitive load" obteniendo un total de 397 documentos cuya distribución era de la siguiente manera: Web of Science (135), Scopus (98), Mediline (33), PsyInfo (112) y Psychology and Behavioral Sciences Collection (19).

Dado el inmenso número de artículos se redujeron estableciendo los siguientes criterios de cribado con el fin de que estuvieran lo más relacionados posible con el tema tratado, que consistieran en hallazgos recientes y que fueran artículos accesibles para el autor, pudiendo ser de acceso restringido pero accesible por estar suscrito a algún servicio de la Universidad de Salamanca o que directamente fuesen de acceso abierto. Se establecieron pues los siuientes

- Que incluyeran las dos palabras de búsqueda den título, resumen o palabras clave ("cognitive load" AND "deception").

- Publicados en los últimos 5 años (desde 2015 incluido).

- Texto completo disponible.

De esta manera se obtuvieron 54 encontrándose en Web of Science (21), Scopus (11), Mediline (3), PsyInfo (13) y Psychology and Behavioral Sciences Collection (6). Con este filtrado se procedió al análisis y lectura de los abstract de todos ellos y se seleccionaron los que se adecuaban al objeto de este trabajo bajo las siguientes condiciones:

- Que no estuvieran repetidos.

- Que consistieran en trabajos empíricos.

- Que se basaban en técnicas de carga cognitiva inducida.

Con el segundo criterio se descartaron aquellos que consistían en propuestas o revisiones teóricas relacionadas con este ámbito o metaanálisis. Con el último criterio se excluyeron algunos en los que existía carga cognitiva, pero esta se debía a la situación (ej. pacientes terminales). También se excluyeron aquellos en los que la carga cognitiva se debía al lenguaje (ej. hacer la entrevista en un idioma no nativo) por considerarse que tiene una implicación distinta en un ámbito de detección del engaño y de difícil aplicación. Es decir, en una entrevista de investigación es relativamente sencillo pedirle al entrevistado que haga una tarea durante la entrevista o que cuente el relato en orden inverso, pero no que la haga en otro idioma porque no todo el mundo está capacitado para hacerlo. Tras este último cribado quedaron para su análisis un total de 8 artículos.

La lista de artículos seleccionados puede verse en la tabla 2. De los 8 elegidos finalmente, 3 se publicaron en 2015, 2 en 2016, 2 en 2017 y 1 en 2018, lo que indica el interés por esta materia en los últimos años, si bien hay que tener en cuenta que ha habido otros muchos más artículos en este período, pero descartados por los motivos antes expuestos.

En cuanto a las revistas de impacto donde han sido publicados destaca Frontiers in Psychology con 4, y Plos One con 2, mientras que el resto tienen uno cada una: Legal and Criminological Psychology, Applied Cognitive Psychology, Psychiatry, Psychology and Law y Applied Psychophysiology and Biofeedback.

\section{Resultados}

Los diversos estudios aquí analizados usan distintas técnicas para inducir la carga cognitiva. En el de Dando et al. (2015) se busca hacerlo a partir de manipular el momento en el que se le facilita la información que se tiene al entrevistador de manera que en función del momento en que esta se de aumentará más o menos la carga cognitiva. En Diana et al. (2018) se basa en una tarea simultánea de recuerdo de frases, donde además de mentir tienen que recordar una lista de frases numeradas. En Fenn et al. (2015) es a través del orden inverso en intención futura, donde tienen se les pregunta por una tarea que harán en el futuro. Gawrylowicz et al. (2016) realizan una tarea simultánea de simulación de conducción mientras son preguntados. Li et al. (2018) por su parte crean una situación de simulación de un ordenador que detecta la mentira y al que tienen que engañar. También Liu et al. (2017) lo hacen con una prueba de memoria donde tienen que reconocer estímulos en pantalla y recordad su localización. Saykaly et al. (2017) lo hacen a través de un interrogatorio al igual que Ströfer et al. (2016), con la diferencia de que en el primero hay una evaluación previa al interrogatorio. 
Cabezas, S. (2019). Revisión de las técnicas de carga cognitiva inducida en el ámbito de la detección de la mentira. Behavior \& Law Journal, 5(1), 40-51.

Tabla 2. Listado de artículos seleccionados.

\begin{tabular}{|c|c|c|c|}
\hline Autores & $\begin{array}{c}\text { Año de } \\
\text { publicación }\end{array}$ & Título & Revista \\
\hline $\begin{array}{l}\text { Dando, Bull, Ormerod, \& } \\
\text { Sandham }\end{array}$ & 2015 & $\begin{array}{l}\text { Helping to sort the liars from the truth-tellers: The gradual revelation } \\
\text { of information during investigative interviews }\end{array}$ & $\begin{array}{l}\text { Legal and Criminological } \\
\text { Psychology }\end{array}$ \\
\hline $\begin{array}{l}\text { Diana, B., Zurloni, V., Elia, M., } \\
\text { Cavalera, C., Realdon, O., } \\
\text { Jonsson, G. K., \& Anguera, M. } \\
\text { T. }\end{array}$ & 2018 & $\begin{array}{l}\text { T-Pattern Analysis and Cognitive Load Manipulation to Detect Low- } \\
\text { Stake Lies: An Exploratory Study }\end{array}$ & Frontiers in Psychology \\
\hline $\begin{array}{l}\text { Fenn, E., McGuire, M., } \\
\text { Langben, S., \& Blandón-Gitlin, }\end{array}$ & 2015 & $\begin{array}{l}\text { A reverse order interview does not aid deception detection regarding } \\
\text { intentions }\end{array}$ & Frontiers in Psychology \\
\hline $\begin{array}{l}\text { Gawrylowicz, J., Fairlamb, S., } \\
\text { Tantot, E., Qureshi, Z., Redha, } \\
\text { A., \& Ridley, A. M }\end{array}$ & 2016 & $\begin{array}{l}\text { Does practice make the perfect liar? The effect of rehearsal and } \\
\text { increased cognitive load on cues to deception. }\end{array}$ & $\begin{array}{l}\text { Applied Cognitive } \\
\text { Psychology }\end{array}$ \\
\hline $\begin{array}{l}\text { Li, F., Zhu, H., Xu, J., Gao, Q., } \\
\text { Guo, H., Wu, S., Li., X. \& He, } \\
\text { S. }\end{array}$ & 2018 & $\begin{array}{l}\text { Lie detection using fNIRS monitoring of inhibition-related brain } \\
\text { regions discriminates infrequent but not frequent liars }\end{array}$ & Frontiers in Psychology \\
\hline $\begin{array}{l}\text { Liu, Y., Wang, C., Jiang, H., } \\
\text { He, H., \& Chen, F }\end{array}$ & 2017 & $\begin{array}{l}\text { Lie construction affects information storage under high memory load } \\
\text { condition }\end{array}$ & Plos One \\
\hline $\begin{array}{l}\text { Saykaly, C., Crossman, A., \& } \\
\text { Talwar, V. }\end{array}$ & 2017 & $\begin{array}{l}\text { High Cognitive Load During Cross-Examination: Does It Improve } \\
\text { Detection of Children's Truths and Lies?. }\end{array}$ & $\begin{array}{l}\text { Psychiatry, Psychology and } \\
\text { Law }\end{array}$ \\
\hline $\begin{array}{l}\text { Ströfer, S., Noordzij, M. L., } \\
\text { Ufkes, E. G., \& Giebels }\end{array}$ & 2015 & $\begin{array}{l}\text { Deceptive intentions: Can cues to deception be measured before a lie } \\
\text { is even stated? }\end{array}$ & Plos One \\
\hline $\begin{array}{l}\text { Ströfer, S., Uflkes, E. G., } \\
\text { Noordzij, M. L., \& Giebels, E }\end{array}$ & 2016 & $\begin{array}{l}\text { Catching a deceiver in the act: Processes underlying deception in an } \\
\text { interactive interview setting }\end{array}$ & $\begin{array}{l}\text { Applied Psychophysiology } \\
\text { and Biofeedback }\end{array}$ \\
\hline
\end{tabular}

Estas técnicas se podrían agrupar en aquellas que tienen que ver con una tarea de manera simultánea (Tarea simultánea de recuerdo de frases, Simulador de conducción, Tarea de memoria) o mediante los factores propios derivados del estrés de la entrevista/situación (Manipulando el momento de la revelación de la información, Orden inverso en intención futura, Simulación de detección de engaño, Interrogatorio). Resultan especialmente interesantes este último grupo pues es más similar al que nos encontraríamos en un entorno habitual de detección de la mentira.

Los resultados de estas técnicas podemos verlas en la tabla 4. En los estudios de tarea simultánea como Diana et al. (2015) encuentran que en análisis de conductas de los entrevistados a los que se les ha aumentado la carga cognitiva tuvieron más conductas de tocarse a sí mismos. Dentro de este grupo destaca que los sinceros se da más el control de gestos mientras que en los mentirosos predominaban los gestos rítmicos; con los patrones-T ni hay diferencias con el grupo control, pero dentro del grupo experimental destaca el número de patrones únicos.

En Gawrylowicz et al. (2016), donde se realiza la tarea de conducción, no encontraron diferencias entre los sinceros y los mentirosos ensayados, pero sí con los no ensayados. Los tiempos de reacción aumentaron un poco con la entrevista. Todo ello se tradujo en que para los sinceros las preguntas eran más fáciles y tenían mayor confianza en que les creyeran.
El último de tarea simultánea, el de Liu et al. (2017), se vio que aumentaba significativamente la actividad de retardo contralateral en la situación de la tarea de memoria con alta carga cognitiva, mientras que con baja carga no había apenas diferencias entre decir la verdad y mentir. De nuevo, parece evidenciar que mentir requiere recursos cognitivos que no están disponibles si se usan en otra tarea.

En cuanto a los estudios de factores derivados de la entrevista y estrés/situación tenemos el de Dando et al. (2015) donde el momento de revelar la información afectó a los niveles de carga cognitiva, produciendo el mayor efecto en los mentirosos al revelarse de manera gradual, algo a tener en cuenta en los interrogatorios policiales. La forma de revelar la información solo ayudó al juicio de los observadores cuando esta se hizo progresivamente.

En el estudio de Fenn et al. (2015) lo que se estudiaba era la intención, encontrando que el orden inverso no ayudó mucho, aunque sí hizo identificar mejor a los mentirosos pero empeoró la detección de los sinceros respecto al orden secuencial y derivó en mayor número de juicios de engaño. Las claves de comportamiento tampoco mejoraron mucho la precisión, haciendo que los que decían la verdad mostraran más comportamientos de engaño. 
Tabla 3. Descripción de los estudios analizados.

\begin{tabular}{|c|c|c|c|}
\hline Artículo & Participantes & Aumento CC & Descripción \\
\hline Dando et al. (2015) & $\begin{array}{l}151 \\
\text { entrevistado y } \\
13 \\
\text { observadores }\end{array}$ & $\begin{array}{l}\text { Manipulando el } \\
\text { momento de la } \\
\text { revelación de la } \\
\text { información }\end{array}$ & $\begin{array}{l}\text { Los entrevistados se sometían a una entrevista en la que la mitad debían decir la verdad y } \\
\text { la otra mitad mentir. Existían tres condiciones en las cuales el entrevistador revelaba la } \\
\text { información que sabía al principio de la entrevista, al final o de forma gradual. } \\
\text { Posteriormente, un tercer observador tenía que ver la entrevista y emitir un juicio sobre } \\
\text { si el entrevistado decía la verdad o no. Después se le pasaba al entrevistado un } \\
\text { cuestionario para informar sobre su nivel de engaño, demanda cognitiva y motivación }\end{array}$ \\
\hline Diana et al. (2018) & $\begin{array}{l}40 \\
\text { entrevistados }\end{array}$ & $\begin{array}{ll}\text { Tarea } & \\
\text { simultánea } & \text { de } \\
\text { recuerdo } & \text { de } \\
\text { frases } & \end{array}$ & $\begin{array}{l}\text { Los participantes tenían que ver dos segmentos de vídeo y luego decir la verdad sobre } \\
\text { uno y mentir sobre el otro. En el grupo control se les dijo que prestaran especial } \\
\text { atención. El grupo experimental además tenía que recordar una lista de } 4 \text { frases } \\
\text { numeradas y que tendrían que recordarlas cuando el experimentador se las pida en } \\
\text { cualquier momento. Durante la entrevista se analizó y categorizó las conductas de los } \\
\text { entrevistados y posteriormente se hizo un análisis de patrones-T. }\end{array}$ \\
\hline Fenn et al. (2015) & $\begin{array}{l}19 \\
\text { entrevistados } \\
\text { y } \quad 157 \\
\text { observadores } \\
\text { (exp. 1) y } 91 \\
\text { (exp. 2) }\end{array}$ & $\begin{array}{l}\text { Orden inverso } \\
\text { en intención } \\
\text { futura }\end{array}$ & $\begin{array}{l}\text { A diferencia de otros se centra en la intención futura. Los participantes planean hacer } \\
\text { una tarea, pero son interrumpidos y se les entrevista acerca de sus intenciones. A un } \\
\text { grupo se entrevista de manera secuencial y a otro en orden inverso. La mitad dice la } \\
\text { verdad sobre sus intenciones y la otra mitad no. Luego dos grupos de observadores debe } \\
\text { evaluar si dicen la verdad o no según su criterio (experimento 1) o según } 10 \text { claves de } \\
\text { comportamiento que se les indican (experimento } 2 \text { ). }\end{array}$ \\
\hline $\begin{array}{l}\text { Gawrylowicz et al. } \\
\text { (2016) }\end{array}$ & 90 & $\begin{array}{l}\text { Simulador de } \\
\text { conducción }\end{array}$ & $\begin{array}{l}\text { Los participantes tenían que hacer o imaginar cuatro actividades. También realizar una } \\
\text { prueba en un simulador de conducción y percepción de riesgos Después se les hacía una } \\
\text { entrevista sobre ello mientras que estaban en el simulador y posteriormente se les pasó } \\
\text { un cuestionario preguntándoles qué tal difícil les había parecido la tarea de conducción. } \\
\text { Se hicieron tres grupos: sinceros, mentirosos ensayados y mentirosos no ensayados. Se } \\
\text { determinó quién mentía a partir de ocho medidas de claves vocales y verbales. }\end{array}$ \\
\hline Li et al. (2018) & $\begin{array}{l}32 \\
\text { participantes }\end{array}$ & $\begin{array}{ll}\text { Simulación } & \text { de } \\
\text { detección } & \text { de } \\
\text { engaño } & \end{array}$ & $\begin{array}{l}\text { Los sujetos tenían que responder un cuestionario de } 64 \text { preguntas autorelacionadas con } \\
\text { veracidad. Se asignaron al grupo inocentes y mentirosos. En la condición inicial todos } \\
\text { tuvieron que responder a las preguntas de manera veraz. En la condición de tarea (una } \\
\text { simulación de detección de engaño por un ordenador) a los inocentes se les dijo que } \\
\text { debían ser sinceros y mostrarse con veracidad. A los del grupo mentirosos se les dijo que } \\
\text { tenían que ocultar su identidad y convencer al ordenador, si no perdían el dinero, y } \\
\text { podían decir tantas mentiras como quisieran;, en función el número de mentiras se } \\
\text { crearon dos subgrupos: mentirosos frecuentes y mentirosos poco frecuentes. Se usó } \\
\text { espectroscopia funcional de infrarrojo cercano para monitorizar la inhibición de regiones } \\
\text { cerebrales (EFIC). }\end{array}$ \\
\hline Liu et al. (2017) & $\begin{array}{l}22 \\
\text { participantes }\end{array}$ & $\begin{array}{l}\text { Tarea } \\
\text { memoria }\end{array}$ & $\begin{array}{l}\text { Se les puso delante de un monitor donde se les presentaba un banco de estímulos en la } \\
\text { pantalla con distintas localizaciones y a los participantes se les instruyó que debían dar } \\
\text { una respuesta engañosa al siguiente período de prueba si veían una flecha roja, o dar una } \\
\text { respuesta honesta si veían una flecha verde. Se les mostró un ítem que debían decir si } \\
\text { estaba en el lugar indicado o no, tras haberse presentado la información previamente. } \\
\text { Hubo } 480 \text { ensayos bajo condiciones de baja carga cognitiva y de alta carga cognitiva. Se } \\
\text { registró la actividad cerebral mediante electroencefalograma. }\end{array}$ \\
\hline Saykaly et al. (2017) & $\begin{array}{l}88 \\
\text { participantes }\end{array}$ & Interrogatorio & $\begin{array}{l}\text { Veían un total de ocho vídeos con el testimonio de cuatro niños diferentes que podían } \\
\text { estar diciendo la verdad o no. Se les pidió que determinaran si los relatos de los niños } \\
\text { sobre un evento incluían una negación verdadera, una negación falsa, una aseveración } \\
\text { verdadera o una aseveración falsa sobre un juego jugado durante una visita a casa que } \\
\text { tuvo lugar una semana antes (examen directo). Después se les hacía un interrogatorio a } \\
\text { los niños y se les volvía a preguntar. }\end{array}$ \\
\hline Ströfer et al. (2016) & $\begin{array}{l}63 \\
\text { participantes }\end{array}$ & Interrogatorio & $\begin{array}{l}\text { Se les expuso a una situación en la que firmaban un documento que legalmente no } \\
\text { estaba permitido. Después se les hizo una entrevista donde se les preguntada } \\
\text { directamente por esta conducta mientras se monitorizaba la respuesta eléctrica. } \\
\text { Recibieron recomendaciones sobre cómo comportarse en la entrevista y se distribuyeron } \\
\text { en tres condiciones: verdad, mentira e intención (verdad en todas las preguntas salvo en } \\
\text { lo referente a la firma del documento). Al terminar hacían un cuestionario para evaluar } \\
\text { su nivel de estrés y carga cognitiva. }\end{array}$ \\
\hline
\end{tabular}

Tabla 4. Resultados de los estudios analizados.

\begin{tabular}{|l|l|}
\hline \multicolumn{1}{|c|}{ Artículo } & \multicolumn{1}{c|}{ Resultados } \\
\hline Dando et al. (2015) & $\begin{array}{l}\text { El grupo que mentía experimentó una mayor demanda cognitiva cuando la información se revelaba de manera gradual, } \\
\text { seguida de cuando se revelaba al final y la que menos fue cuando se daba al principio. En el grupo de los que decían la } \\
\text { verdad hubo pocas diferencias significativas. Así mismo, el nivel de engaño que creyeron tener los entrevistados fue más } \\
\text { bajo cuando la información se revelaba de manera gradual. }\end{array}$ \\
& $\begin{array}{l}\text { El nivel de acierto de los observadores al juzgar las entrevistas fue de 50\% para los mentirosos y 48\% para los sinceros } \\
\text { cuando la información se revelaba al principio; de 54\% para los mentirosos y 44\% para los sinceros cuando la información } \\
\text { se revelaba al final; y de } 66 \% \text { para los mentirosos y } 76 \% \text { para los sinceros cuando la información se revelaba gradualmente. }\end{array}$ \\
\hline
\end{tabular}




\begin{tabular}{|c|c|}
\hline Diana et al. (2018) & $\begin{array}{l}\text { Las conductas de tocarse a uno mismo fueron mayores en el grupo experimental. Dentro de los grupos, en el control los } \\
\text { gestos de ilustración fueron más frecuentes en los sinceros, mientras que en el grupo experimental los gestos rítmicos } \\
\text { fueron más frecuentes en los que mentían. En lo demás no hubo diferencias significativas. En el análisis con patrones-T no } \\
\text { se encuentran diferencias en el grupo control, pero sí en el grupo experimental donde el número de patrones únicos es } \\
\text { mayor en los sinceros que en los mentirosos. }\end{array}$ \\
\hline Fenn et al. (2015) & $\begin{array}{l}\text { Experimento 1. Los que dijeron la verdad fueron peor identificados en orden inverso que en secuencial. Los que mentían } \\
\text { fueron algo mejor detectados en la condición de orden inverso que en secuencial, pero no mucho. La introducción de la } \\
\text { entrevista en orden inverso produjo un mayor número de juicios de engaño. } \\
\text { Experimento 2. Los que dijeron la verdad mostraron más comportamientos de engaño y menos de veracidad en orden } \\
\text { inverso, lo que redujo la precisión en el juicio como sinceros, siendo coherente con los resultados del Experimento } 1 \text {. }\end{array}$ \\
\hline $\begin{array}{l}\text { Gawrylowicz et al. } \\
\text { (2016) }\end{array}$ & $\begin{array}{l}\text { Se comprobó el efecto de doble tarea al realizar la simulación de conducción y la entrevista. Hubo diferencias entre los } \\
\text { mentirosos no ensayados y los sinceros, aumentando la velocidad en el caso de los primeros respecto al ensayo inicial y } \\
\text { disminuyendo la de los segundos. No hubo diferencias entre mentirosos ensayados y no ensayados. La entrevista tuvo } \\
\text { menos efecto en los tiempos de reacción de los sinceros. Los sinceros dieron más detalles visuales y auditivos que los } \\
\text { mentirosos ensayados y no ensayados y también proporcionaban menos operaciones cognitivas que los mentirosos } \\
\text { ensayados y no ensayados. Los sinceros percibieron las preguntas de la entrevista como más fáciles que los mentirosos y } \\
\text { también pensaros que era más probable que el entrevistador les creyera. }\end{array}$ \\
\hline Li et al. (2018) & $\begin{array}{l}\text { Los mentirosos infrecuentes exhibían mayores actividades neuronales que los mentirosos frecuentes y los inocentes en el } \\
\text { giroscopio frontal medio izquierdo (GFM) cuando realizaban las tareas de detección de engaños. Mientras realizaban tareas } \\
\text { de detección de engaños, mentirosos infrecuentes mostraron una mayor activación neural en el GFM izquierdo que en la } \\
\text { línea de base, pero mentirosos e inocentes frecuentes no mostraron este patrón de activación neural en ningún área de } \\
\text { regiones cerebrales relacionadas con la inhibición. Los resultados de los análisis individuales mostraron una precisión } \\
\text { aceptable en la detección de mentirosos poco frecuentes, pero una precisión inaceptable en la detección de mentirosos } \\
\text { frecuentes. La monitorización EFIC de las regiones cerebrales relacionadas con la inhibición es factible para detectar a los } \\
\text { mentirosos infrecuentes, para quienes el engaño puede ser más esforzado y, por lo tanto, más fisiológicamente marcado, } \\
\text { pero no a los mentirosos frecuentes. }\end{array}$ \\
\hline Liu et al. (2017) & $\begin{array}{l}\text { Los análisis basados en la amplitud de la actividad de retardo contralateral (CDA) (mide representaciones en memoria de } \\
\text { trabajo) mostraron que la amplitud de la CDA era menor en el proceso de engaño que en el proceso de decir la verdad } \\
\text { bajo la condición de alta carga. En contraste, bajo la condición de baja carga, no se encontró ninguna diferencia en el CDA } \\
\text { entre los procesos de engaño y de decir la verdad. La construcción de mentiras y el almacenamiento de información } \\
\text { compiten por los recursos en la memoria de trabajo; cuando los recursos de la memoria de trabajo disponibles no pueden } \\
\text { satisfacer esta demanda cognitiva, estos recursos son ocupados por el almacenamiento de información y serían consumidos } \\
\text { para la construcción de mentiras. }\end{array}$ \\
\hline Saykaly et al. (2017) & $\begin{array}{l}\text { El interrogatorio de carga cognitiva alta no mejoró las tasas de detección dado que los participantes estaban en el nivel de } \\
\text { azar tanto para el examen directo }(49,4 \%) \text { como para el interrogatorio }(52,3 \%) \text {. La precisión para las historias verdaderas } \\
\text { era mayor que para las historias falsas. El interrogatorio mejoró las tasas de detección de las historias falsas, pero empeoró } \\
\text { la precisión de las historias verdaderas. Sin embargo, los participantes calificaron los informes verdaderos de los niños más } \\
\text { pequeños como más creíbles y veraces que sus informes falsos. Los participantes calificaron los informes falsos de los } \\
\text { niños mayores como más creíbles y veraces que sus informes verdaderos. }\end{array}$ \\
\hline Ströfer et al. (2016) & $\begin{array}{l}\text { Los mentirosos se diferenciaban constantemente de los sinceros en la actividad del sistema nervioso simpático (SNS), la } \\
\text { carga cognitiva auto-reportada y el estrés. A través de todas las entrevistas, la actividad del SNS se correlacionó más } \\
\text { fuertemente con los auto-reportes de carga cognitiva que con el estrés, lo que apoya el enfoque de carga cognitiva. } \\
\text { Además, los mentirosos que decían la verdad y mentían en una sola pregunta crucial (intención), en particular, divergían en } \\
\text { el estrés auto-reportado por los narradores de la verdad. En cuanto a la actividad de la SNS y la carga cognitiva } \\
\text { autoinformada, no se encontraron diferencias. }\end{array}$ \\
\hline
\end{tabular}

Li et al. (2018), creando la situación de simulación de detección de engaño, mostraron eficacia en la medición de actividades neuronales en el giroscopio frontal medio izquierdo, si bien esto sólo era con los mentirosos infrecuentes, dado que con los mentirosos frecuentes no era muy efectivo.

En cuanto al interrogatorio, Saykaly et al. (2017) encontró que un interrogatorio de carga cognitiva alta no mejoró las tasas de detección, dado que aumentó el número de detección de historias falsas pero redujo el de historias verdaderas.

Por último, Ströfer et al. (2016) analizaron la actividad del sistema nervioso simpático existiendo una fuerte correlación con niveles altos de estrés y carga cognitiva. Los mentirosos e los del grupo intención experimentaron mayores niveles de carga cognitiva y estrés.

\section{DISCUSIÓN Y CONCLUSIONES}

Como se comentaba inicialmente los estudios muestran que mentir es cognitivamente más complicado que decir la verdad en consonancia con estudios previos (BlandónGitlin, Fenn, Masip y Yoo, 2014; Masip y Herrero, 2015a; 2015b; Walczyk et al., 2009). De esta manera se han expuesto diversas formas de imponer esa carga cognitiva: Tarea simultánea de recuerdo de frases, Simulador de conducción, Tarea de memoria, Manipulando el momento de la revelación de la información, Orden inverso en intención futura, Simulación de detección de engaño e Interrogatorio. $\mathrm{Si}$ bien la perspectiva es alentadora los 
resultados de estos estudios no lo han sido tanto, dado que como hemos visto tampoco es que hayan supuesto un gran incremento del nivel de aciertos en detección de mentiras, lo que no se diferencia mucho de la gente que cree que es buen detector de mentiras cuando no lo es (Alonso, 2009; Masip, 2005). Igualmente, también puede ser que sea porque se fijaron en las claves incorrectas para hacer los juicios dado que no solemos utilizar claves fiables (Vrij, 2008). Lo que sí parece claro es que los mentirosos tendrán menos recursos cognitivos, como por ejemplo se pone de manifiesto en el estudio de Liu et al. (2017); sin embargo, si esto lo hacen con cierta frecuencia pueden no dar resultados significativos como en el estudio de $\mathrm{Li}$ et al (2018). Se esperaría que al aumentar la demanda cognitiva los mentirosos fueran menos capaces que los sinceros (Vrij et al., 2017), sin embargo, no se encuentran diferencias significativas.

Investigadores previamente (Vrij et al., 2008; Vrij, Leal, Mann y Fisher, 2012) habían determinado que una narración en orden inverso hace modificar la conducta del entrevistado otorgando mayores indicadores para discriminar entre verdades y mentiras; sin embargo, en el estudio de Fenn et al. (2015) lo que se encontró es que, sin atender a las claves de comportamiento, el orden inverso daba más falsos positivos y apenas mejoró la detección de orden inverso. Se esperaría que si se fijaran en claves de comportamiento aumentara su eficacia, pero lo cierto fue que tampoco fue así, dado que los que eran sinceros mostraron más comportamiento de engaños y menos de veracidad en orden inverso, lo que sugiere una revisión de este planteamiento. Puede ser, como sugieren los autores, que determinadas entrevistas con altar carga cognitiva aumenten también el nivel de falsos positivos en inocentes. Si bien, cabe destacar que en este experimento se hacía referencia a una intención futura, no a una experiencia pasada que había ocurrido, la cual podría variar los resultados (Vrij, Leal, Mann y Fisher, 2012).

Continuando con esta idea de analizar el comportamiento como base para determinar si una persona miente o no (Ovejero, 2009) se analizaron también en el estudio de Diana et al. (2018), si bien algunas de las analizadas mostraron cierta correlación, tampoco fueron determinantes a nivel global. Mejores resultados dio el estudio de Gawrylowicz et al. (2016) donde sí se mostró que los sinceros les resultó más fácil realizar la entrevista y sus tiempos de reacción apenas aumentaron, sin bien el número de detalles y las operaciones cognitivas sí fueron más determinantes. En definitiva, la conducta no verbal se ha usado como una fuente de detección de mentiras en otros ámbitos (López, León y Grau, 2016; Valverde et al., 2013) pero aquí no parece haber resultado muy relevantes.

En lo referente a los interrogatorios se ha mostrado que técnicas como mantener contacto visual con el entrevistador (Vrij, Mann, Leal y Fisher, 2010) o mediante turnos forzados que pueden emplearse cuando se entrevistan dos o más entrevistados al mismo tiempo (Vernham, Vrij, Mann, Leal y Hillman, 2014). En los estudios aquí analizados vemos que el aumentar la carga cognitiva en estos interrogatorios no mejoró significativamente la tasa de acierto. En el estudio de Saykaly et al. (2017) apenas experimentó mejora en la tasa de acierto, si bien es cierto que al ser con niños los resultados pueden no poder extrapolarse a población adulta. En el de Ströfer et al. (2016) sí se encontró fuerte relación entre carga cognitiva y mentira, pero en este caso no se evaluaba la veracidad de la declaración.

Por último, la técnica que pareció dar los mejores resultados fue la de manipulación del momento en que se da la información, con tasas de acierto del 70\% aproximadamente cuando se revelaba de forma gradual. Aun así, estas tasas son similares a las de otras técnicas como el CBCA o RM (Gödert, et al., 2005; Lee et al., 2008; Vrij, 2000, 2005). Estudios previos mostraron más eficacia del enfoque cognitivo de la evaluación de la credibilidad que del enfoque tradicional (Vrij, Fisher, Blank, Leal y Mann, 2016; Vrij. Fisher y Blank, 2017) pero a la vista de estos resultados requieres estudios más profundos y un mayor desarrollo, si bien es cierto que todavía se está explorando su potencial.

\section{REFERENCIAS}

Alonso, H. (2009). Los policías como detectores del engaño: investigación en torno al efecto de sesgo del investigador (Tesis doctoral). Universidad de Salamanca, Salamanca, España.

Ansarra, R., Colwell, K., Hiscock-Anisman, C., Hines, A., Fleck, R., Cole, L., \& Belarde, D. (2011). Augmenting acid with affective details to assess credibility. European Journal of Psychology Applied to Legal Context, 3(2), 141-158.

Ben-Shakhar, G., y Elaad, E. (2003). The validity of psychophysiological detection of information with the Guilty Knowledge Test: A metaanalytic review. Journal of Applied Psychology, 88(1), 131-151.

Blandón-Gitlin, I., Fenn, E., Masip, J. y Yoo, A. H. (2014). Cognitiveload approaches to detect deception: searching for cognitive mechanisms. Trends in Cognitive Sciences, 18(9), 441-444.

Blandón-Gitlin, I., Pezdek, K., Rogers, R. y Brodie, L. (2005). The effects of event familiarity on criterion-based content analysis ratings: An experimental study. Law and Human Behavior, 29, 187- 
Cabezas, S. (2019). Revisión de las técnicas de carga cognitiva inducida en el ámbito de la detección de la mentira. Behavior \& Law Journal, 5(1), 40-51.

197.

Bogaard, G., Meijer, E. H., \& Vrij, A. (2014). Using an example statement increases information but does not increase accuracy of CBCA, RM, and SCAN. Journal of Investigative Psychology and Offender Profiling, 11(2), 151-163.

Bond, C. F. y DePaulo, B. M. (2006). Accuracy of deception judgments. Personality and Social Psychology Review, 10, 214-234. doi:10.1207/s15327957pspr1003_2.

Colwell, K., Hiscock, C. K., \& Memon, A. (2002). Interviewing techniques and the assessment of statement credibility. Applied Cognitive Psychology: The Official Journal of the Society for Applied Research in Memory and Cognition, 16(3), 287-300.

Dando, C. J., Bull, R., Ormerod, T. C., \& Sandham, A. L. (2015). Helping to sort the liars from the truth-tellers: The gradual revelation of information during investigative interviews. Legal and Criminological Psychology, 20(1), 114-128.

DePaulo, B. M., Lindsay, J. J., Malone, B. E., Muhlenbruck, L., Charlton, K. y Cooper, H. (2003). Cues to deception. Psychological Bulletin, 129, 74-118. doi:10.1037/0033- 2909.129.1.74.

Diana, B., Zurloni, V., Elia, M., Cavalera, C., Realdon, O., Jonsson, G. K., \& Anguera, M. T. (2018). T-Pattern Analysis and Cognitive Load Manipulation to Detect Low-Stake Lies: An Exploratory Study. Frontiers in psychology, 9, 257.

Fenn, E., McGuire, M., Langben, S., \& Blandón-Gitlin, I. (2015). A reverse order interview does not aid deception detection regarding intentions. Frontiers in psychology, 6, 1298.

Garrido, V y Sobral, J. (2008). La Investigación Criminal: psicología aplicada al descubrimiento, captura y condena de los criminales. Barcelona: Nabla.

Gawrylowicz, J., Fairlamb, S., Tantot, E., Qureshi, Z., Redha, A., \& Ridley, A. M. (2016). Does practice make the perfect liar? The effect of rehearsal and increased cognitive load on cues to deception. Applied Cognitive Psychology, 30(2), 250-259.

Gödert, H. M., Gamer, M., Rill, H. G., y Vossel, G. (2005). Statement validity assessment: Inter-rater reliability of criteria-based content analysis in the mockcrime paradigm. Legal and Criminological Psychology, 10, 225-245.

Godoy-Cervera, V. e Higueras, L. (2005). El análisis de contenido basado en criterios (CBCA) en la evaluación de la credibilidad del testimonio. Papeles del psicólogo, 26(92).

Granhag, P. A., Strömwall, L. y Hartwig, M. (2007). The SUEtechnique: The way to interview to detect deception. Forensic Update, $88,25-29$.

Hartwig, M., Granhag, P. A., Strömwall, L. A. y Vrij, A. (2005). Detecting deception via strategic disclosure of evidence. Law and Human Behavior, 29, 469-484.

Hartwig, M., Granhag, P. A., Strömwall, L. A., y Kronkvist, O. (2006). Strategic use of evidence during police interviews: When training to detect deception works. Law and Human Behavior, 30(5), 603-619.

Hauch, V., Blandón-Gitlin, I., Masip, J. y Sporer, S. L. (2015). Are computers effective lie detectors? A meta-analysis of linguistic cues to deception. Personality and Social Psychology Review, 19(4), 307-342.

Jiménez, J. (2015). Psicología e Investigación Criminal. Psicología Criminalista. Pamplona: Lex Nova.

Johnson, P. (2006). Corporate Strategy: Purpose. In Astute Competition (pp. 141-155). Emerald Group Publishing Limited.
Kleiner, M. (2002). Handbook of polygraph testing. Londres: Academic Press.

Köhnken, G., Manzanero, A. L. y Scott, M. T. (2015). Análisis de la validez de las declaraciones: mitos y limitaciones. Anuario de Psicología Jurídica, 25, 13-19.

Lavoie, J.A.A. (2004) The efficacy of CBCA and RM in Discriminating Between Reports of Single, Repeated, and Fabricated Events. Simon Fraser University, Burnaby, Canadá.

Lee, Z., Klaver, J. R. y Hart, S. D. (2008). Psychopathy and verbal indicators of deception in offenders. Psychology, Crime and Law, 14(1), 73-84.

Li, F., Zhu, H., Xu, J., Gao, Q., Guo, H., Wu, S., Li, X., \& He, S. (2018). Lie detection using fNIRS monitoring of inhibition-related brain regions discriminates infrequent but not frequent liars. Frontiers in buman neuroscience, 12, 71 .

Liu, Y., Wang, C., Jiang, H., He, H., \& Chen, F. (2017). Lie construction affects information storage under high memory load condition. PloS one, 12(7), e0181007.

López, R., León, F. y Grau, M. (Coords.). (2016). Comportamiento no verbal: más allá de la comunicación y el lenguaje. Madrid: Pirámide.

MacLaren, V. V. (2001). A quantitative review of the Guilty Knowledge Test. Journal of Applied Psychology, 86(4), 674-683.

Mann, S., Vrij, A., Shaw, D. J., Leal, S., Ewens, S., Hillman, J., Granhag, P. A. \& Fisher, R. P. (2013). Two heads are better than one? How to effectively use two interviewers to elicit cues to deception. Legal and Criminological Psychology, 18(2), 324-340.

Masip, J. (2005). ¿Se pilla antes a un mentiroso que a un cojo? Sabiduría popular frente a conocimiento científico sobre la detección noverbal del engaño. Papeles del Psicólogo, 92(26), 78-91.

Masip, J. y Alonso, H. (2006). Verdades, mentiras y su detección: aproximaciones verbales y psicofisiológicas. En E. Garrido, J. Masip y C. Herrero. (Coord.). Psicología Jurídica (pp. 507-558). Madrid: Pearson.

Masip, J. y Garrido, E. (2010). Is There A Kernel Of Truth In Judgements Of Deceptiveness? Anales de Psicologia, 17(1), 101-120.

Masip, J. y Herrero, C. (2015a). Nuevas aproximaciones en detección de mentiras I. Antecedentes y marco teórico. Papeles del Psicólogo, 36(2), 83-95.

Masip, J. y Herrero, C. (2015b). Nuevas aproximaciones en detección de mentiras II. Estrategias activas de entrevista e información contextual. Papeles del Psicólogo, 36(2), 96-108.

Masip, J., Sporer, S. L., Garrido, E. y Herrero, C. (2005). The detection of deception with the reality monitoring approach: A review of the empirical evidence. Psychology, Crime \& Law, 11(1), 99-122.

Nahari, G. (2018). Reality Monitoring in the Forensic Context: Digging Deeper into the Speech of Liars. Journal of Applied Research in Memory and Cognition. doi: 10.1016/j.jarmac.2018.04.003.

Ovejero, A. (2009). Fundamentos de psicología jurídica e investigación criminal. Salamanca: Ediciones Universidad de Salamanca.

Sáiz, D., Baqués, J. y Sáiz, M. (2006). Psicología del testigo: conceptos fundamentals. En M. A. Soria y D. Saíz (Coord.). Psicología criminal (pp.123-162). Madrid: Pearson.

Saykaly, C., Crossman, A., \& Talwar, V. (2017). High Cognitive Load During Cross-Examination: Does It Improve Detection of Children's Truths and Lies?. Psychiatry, Psychology and Law, 24(2), 278-

Fundación Universitaria Behavior \& Law $\mid 50$ 
Cabezas, S. (2019). Revisión de las técnicas de carga cognitiva inducida en el ámbito de la detección de la mentira. Behavior \& Law Journal, 5(1), 40-51.

291.

Sporer, S.L. (2004). Reality Monitoring and the Detection of Deception. En Pär Anders Granhag y Leif A. Strömwall (Eds.). The Detection of Deception In Forensic Contexts (pp.64-80). UK: Cambrige University Press.

Steller, M. y Koehnken, G. (1989). Criteria-based statement analysis. En D. C. Raskin (Ed.). Psychological methods in criminal investigation and evidence (pp. 217-245). New York, NY, US: Springer Publishing Co.

Ströfer, S., Ufkes, E. G., Noordzij, M. L., \& Giebels, E. (2016). Catching a deceiver in the act: Processes underlying deception in an interactive interview setting. Applied psychophysiology and biofeedback, 41(3), 349-362.

Valverde, M. J., Ruiz, J. A. y Llor, B. (2013). Valoración de la credibilidad del testimonio: aplicación del modelo Reality Monitoring. Revista internacional de Psicología, 12(02).

Vernham, Z., Vrij, A., Mann, S., Leal, S., \& Hillman, J. (2014). Collective interviewing: Eliciting cues to deceit using a turn-taking approach. Psychology, Public Policy, and Law, 20(3), 309.

Vilariño, M., Novo, M. y Seijo, D. (2011). Estudio de la eficacia de las categorías de realidad del testimonio del Sistema de Evaluación Global (SEG) en casos de violencia de género. Revista Iberoamericana de Psicología y Salud, 2(1), 1-26.

Visu-Petra, G., Varga, M., Miclea, M., \& Visu-Petra, L. (2013). When interference helps: increasing executive load to facilitate deception detection in the concealed information test. Frontiers in psychology, 4, 146.

Vrii, A. (2000). Detecting lies and deceit: the psychology of lying and the implications for professional practice. Chichester: John Wiley \& Sons.

Vrij, A. (2005). Cooperation of liars and truth tellers. Applied Cognitive Psychology, 19, 39-50. doi:10.1002/acp.1050.

Vrij, A. (2008). Detecting Lies and Deceit: Piffalls and Opportunities. Chichester: John Wiley \& Sons.
Vrij, A., Akehurst, L., Soukara, S. y Bull, R. (2004a). Detecting deceit via analysis o verbal and nonverbal behaviour in children's and adults. Human Communication Research, 30, 8-41.

Vrij, A., Akehurst, L., Soukara, S. y Bull, R. (2004b). Let me Inform You How to Tell a Convincing Story: CBCA and Reality Monitoring Scores as a Function of Age, Coaching and Deception. Canadian Journal of Behavioural Science, 36(2), 113-126.

Vrij, A., Edward, K., Roberts, K. y Bull, R. (2000). Detecting deceit via analysis of verbal and nonverbal behaviour. Journal of Nonverbal Behaviour, 24, 239-263.

Vrij, A., Fisher, R. y Blank, H. (2017). A cognitive approach to lie detection: A meta-analysis. Legal and Criminological Psychology, 22, 121. doi: $10.1111 /$ lcrp.12088.

Vrij, A., Fisher, R., Blank, H., Leal, S. y Mann, S. (2016). A cognitive approach to elicit nonverbal and verbal cues of deceit. En J. W. van Prooijen y P. A. M. van Lange (Eds.), Cheating, corruption, and concealment: The roots of dishonest behavior (pp. 284-310). Cambridge, UK: Cambridge University Press.

Vrij, A., Leal, S., Granhag, P. A., Mann, S., Fisher, R., Hillman, J. y Sperry, K. (2009). Outsmarting the liars: The benefit of asking unanticipated questions. Law and Human Behavior, 33, 159-166.

Vrij, A., Mann, S. A., Fisher, R. P., Leal, S. Milne, R. y Bull, R. (2008). Increasing Cognitive Load to Facilitate Lie Detection: The Benefit of Recalling an Event in Reverse Order. Law Human Behavior, 32, 253-265. doi: 10.1007/s10979-007-9103-y.

Vrii, A., Mann, S., Leal, S. y Fisher, R. (2012). Is anyone there? Drawings as a tool to detect deceit in occupation interviews. Psychology, Crime \& Law, 18, 377-388.

Walczyk, J. J., Mahoney, K. T., Doverspike, D. y Griffith-Ross, D. A. (2009). Cognitive lie detection: Response time and consistency of answers as cues to deception. Journal of Business and Psychology, 24, 3349. 